\title{
Unique RUNXI gene rearrangements in acute myeloid Leukemia (AML)
}

\section{Editorial}

RUNX1 is a sequence-specific DNA-binding protein and encodes the alpha subunit core binding factor, a small family of transcription factors called core-binding factors ( $\mathrm{CBFs}$ ). Due to its important role in hematopoiesis, the deficiency of RUNX1 may block the formation of hematopoietic cells. Chromosomal rearrangements and mutations of the RUNX1 gene at 21q22 region have been reported in de novo and therapy-related acute myelogenous leukemia (AML), myelodysplastic syndrome (MDS) and acute lymphocytic leukemia (ALL). A few of the RUNX1 rearrangements that have been reported include $t(1 ; 21)$, $\mathrm{t}(3 ; 21), \mathrm{t}(8 ; 21), \mathrm{t}(12 ; 21), \mathrm{t}(16 ; 21)$ and $\mathrm{t}(17 ; 21)$.

We report here five cases of AML with variant translocation partners of RUNX1; a pediatric AML with $\mathrm{t}(7 ; 21)$, and four adult secondary AML cases with $\mathrm{t}(10 ; 21), \mathrm{t}(16 ; 21)$, $\operatorname{del}(5 \mathrm{q})$ and $\mathrm{t}(7 ; 21)$, and $\mathrm{t}(5 ; 21)$, respectively. Sequential metaphase FISH with RUNX1 specific probe and Cytogenetics were performed in all cases to identify the partner chromosome in the RUNX1 rearrangements.

\section{Cases}

Case 1: A 9year old male was referred for fever, fatigue, and pancytopenia. Bone marrow analysis revealed hypercellularity with a mixture of myeloblasts and immature monocytes comprising $90 \%$ of cells as determined by both morphology and flow cytometry. Molecular analysis for mutations in FLT3, NMP1 and C-KIT were negative, and FISH revealed rearrangement of RUNX1 locus in the chromosome $7 \mathrm{p} 22$ region. analysis confirmed this finding, revealing a unique translocation:46,XY, t(7;21) (p22;q22). Three adults and one child have been reported in the literature with AML or high grade MDS with $t(7 ; 21)$ involving RUNX1-ubiquitin-specific protease gene (USP42) fusion; however, no consistent prognostic information has emerged from these cases to date. ${ }^{1}$ Our patient had reinduction chemotherapy and is currently in remission, awaiting stem cell transplant.

Case 2: A 58year old male was diagnosed with treatment-related AML following urothelial cell carcinoma. In this case, cytogenetics was normal $(46, \mathrm{XY})$ at diagnosis, but follow up bone marrow analysis at four and eleven months later revealed progressive abnormalities (46XY,del(9)(q13q22)[2]/46,XY[18] and 46,XY, t(10;21)(p13;q22) [12]/46,XY[9], respectively). The latter BM was FLT3+ and also demonstrated RUNX1 rearrangement. The partner gene at the 10p13 region is unknown.

Case 3: A 73year old female was diagnosed with a myeloproliferative disorder. Karyotype revealed 46,XX, t(16;21)(q24;q22)[16]/46,XX with FLT3 positivity. FISH analysis revealed rearrangement of RUNX1 with CBFA2T3 (MTG16) gene locus at 16q24. The majority of the cases with $\mathrm{t}(16 ; 21)$ (CBFA2T3; RUNX1) translocation have been reported in patients with prior treatment for a previous malignancy and occur in the context of MDS/ANLL. ${ }^{2,3}$

\author{
Volume 5 Issue I - 2017 \\ Yenamandra A, Hollis A, Zalepa D, Kapp M \\ Department Pathology, Microbiology and Immunology, \\ Vanderbilt University Medical Center, USA
}

\begin{abstract}
Correspondence: Ashwini Yenamandra, PhD FCCMG MS (Data Science),Vanderbilt University Medical Center@I00 Oaks 719,Thompson Lane, Nashville, TN 37204, USA, Email Ashwini.yenamandra@vanderbilt.edu
\end{abstract}

Received: October 04, 2017| Published: October 10, 2017

Case 4: A 61year old female with cardiomyopathy was diagnosed with AML secondary to underlying myelodysplasia. The bone marrow was hypercellular with increased blasts of two distinct populations; one with myeloblasts and the second with monocytic features. The bone marrow was negative for FLT3, NPM1, CEBPA, IDH1, IDH2, DNMT3 and KIT mutations. Cytogenetics revealed a deletion in the long arm of chromosome 5 and a $t(7 ; 21)$ translocation, with a karyotype $46, X X$, $\operatorname{del}(5)(\mathrm{q} 22 \mathrm{q} 35), \mathrm{t}(7 ; 21)(\mathrm{p} 22 ; \mathrm{q} 22)[13] / 46, X X[7]$. About 10 cases were reported in the literature with del $(5 q)$ and $t(7 ; 21)$ suggesting a nonrandom association between the two abnormalities. These cases appear to have a specific immunophenotype and monocytic differentiation with poor prognosis. ${ }^{4}$

Case 5: A 71year old female previously diagnosed with FLT3+AML was referred for status post induction day 14 bone marrow. Bone marrow morphology revealed $35 \%$ myeloblasts and $20 \%$ monocytes, Cytogenetics and FISH revealed abnormal karyotype with RUNX1 rearrangement involving $\mathrm{t}(5 ; 21)$ translocation $(46, \mathrm{XX}, \mathrm{t}(5 ; 21)$ (q35;q22)[7]/46,XX[3]). Next generation studies done outside our institution revealed DNMT3A, IDH2 and RUNX1 mutations. Prognostic significance of these findings is unclear.

We present here five cases with variant RUNX1 translocations involving different chromosome regions in both pediatric (primary) and adult (secondary) AML indicating the importance of the regulatory role of RUNX1 gene in hematopoiesis. Morphology of these cases was unique with myeloblasts and monocytes. The prognosis of the cases we described here is unclear and vary from inconsistent to poor. The subtle RUNX1 rearrangements combined with the unique morphology and inconsistencies of the prognosis makes such cases worth reporting. Genetic testing of new AML cases with RUNX1 FISH probe offers the possibility of identification of additional cases with subtle rearrangements or identification of new partner genes of RUNX1 locus. ${ }^{5-8}$ This will enhance the understanding of the prognosis of these rare cases and may ultimately help in the design of a highly effective therapeutic treatment plan. 

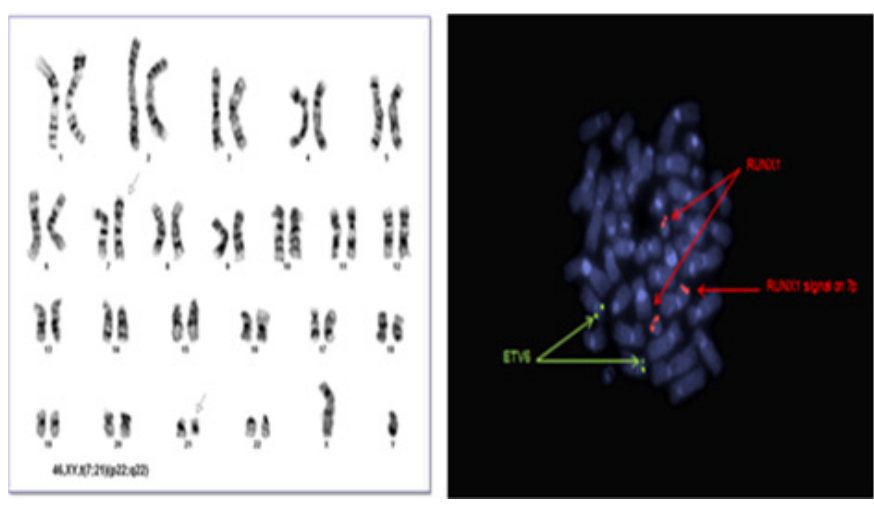

Case I With t(7;2I) Translocation, Karyotype and FISH with RUNXI probe.
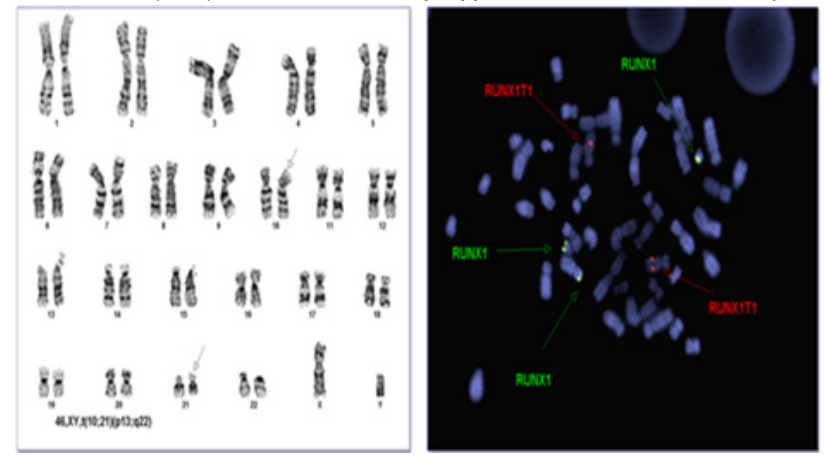

Case 2 With $\mathrm{t}(10 ; 2 I)$ Translocation, Karyotype and FISH with RUNXI probe.

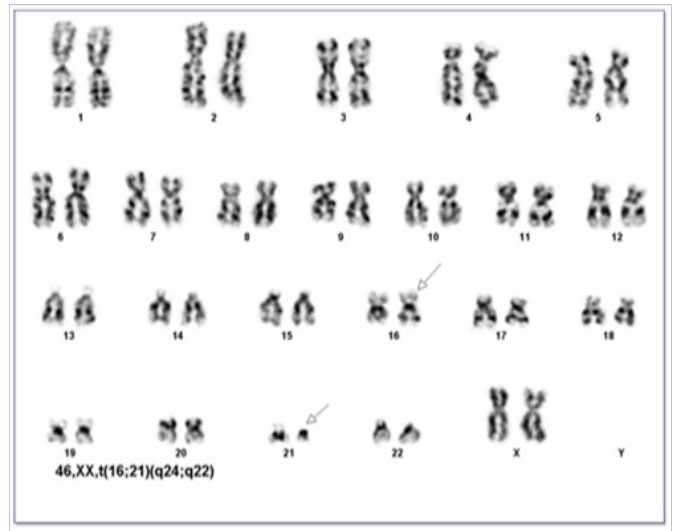

Case 3 Karyotype with $\mathrm{t}(16 ; 21)$ translocation.

\section{Acknowledgements}

None.

\section{Conflict of interest}

The author declares no conflict of interest.

\section{References}

1. Foster N, Paulsson K, Sales M, et al. Molecular characterisation of a recurrent, semi-cryptic RUNX1 translocation $\mathrm{t}(7 ; 21)$ in myelodysplastic syndrome and acute myeloid leukaemia. Br J Haematol. 2010;148(6):938943

2. La Starza R, Sambani C, Crescenzi B, et al. AML1/MTG16 fusion gene from a $\mathrm{t}(16 ; 21)(\mathrm{q} 24 ; \mathrm{q} 22)$ translocation in treatment-induced leukemia after breast cancer. Haematologica. 2001;86(2):212-213.

3. Park IJ, Park JE, Kim HJ, et al. Acute myeloid leukemia with t(16;21) (q24;q22) and eosinophilia: case report and review of the literature. Cancer Genet Cytogenet. 2010;196(1):105-108.

4. Ji J, Loo E, Pullarkat S, et al. Acute myeloid leukemia with $\mathrm{t}(7 ; 21)$ (p22;q22) and 5q deletion: a case report and literature review. Exp Hematol Oncol. 2014;3:8.

5. Swiers G, de Bruijn M, Speck NA. Hematopoietic stem cell emergence in the conceptus and the role of Runx1. Int J Dev Biol. 2010;54(6-7):11511163.

6. De Braekeleer E, Ferec C, De Braekeleer M. RUNX1 translocations in malignant hemopathies. Anticancer Res. 2009;29(4):1031-1037.

7. Liakhovitskaia A, Rybtsov S, Smith $\mathrm{T}$, et al. Runx 1 is required for progression of CD41+ embryonic precursors into HSCs but not prior to this. Development. 2014;141(17):3319-3323

8. Zagaria A, Anelli L, Coccaro N, et al. 5'RUNX1-3'USP42 chimeric gene in acute myeloid leukemia can occur through an insertion mechanism rather than translocation and may be mediated by genomic segmental duplications. Mol Cytogenet. 2014;7:66. 